\title{
Biomimetic silica nanocapsules for tunable sustained release and cargo protection
}

Guang-Ze Yang, ${ }^{a}$ David Wibowo, ${ }^{a}$ Jung-Ho Yun, ${ }^{b}$ Lianzhou Wang, ${ }^{a, b}$ Anton P.J. Middelberg, ${ }^{a}$ and Chun-Xia Zhao ${ }^{a, *}$

${ }^{a}$ Australian Institute for Bioengineering and Nanotechnology, The University of Queensland, St Lucia, QLD 4072, Australia

${ }^{\mathrm{b}}$ School of Chemical Engineering, The University of Queensland, St Lucia, QLD 4072, Australia

Submitted to Langmuir 


\section{KEYWORDS}

Silica nanocapsule; Biosilicification; Sustained release; Release kinetics; Sunlight shielding

\section{ABSTRACT}

Silica nanocapsules have attracted tremendous interest for encapsulation, protection and controlled release of various cargoes due to their unique hierarchical core-shell structure. However, it remains challenging to synthesize silica nanocapsules having high cargo-loading capacity and cargo-protection capability without compromising process simplicity and biocompatibility properties. Here, we synthesized oil-core silica-shell nanocapsules under environmentally friendly conditions by a novel emulsion and biomimetic dual-templating approach using a dual-functional protein, in lieu of petrochemical surfactants, thus avoiding the necessities for the removal of toxic components. A light- and $\mathrm{pH}$-sensitive compound can be facilely encapsulated in the silica nanocapsules with the encapsulation efficiency of nearly $100 \%$. Release of the encapsulated active from the nanocapsules was not shown an indication of undesired burst release. Instead, the release can be tuned by controlling the silica-shell thicknesses i.e., 40 and $77 \mathrm{~nm}$ from which the cargo released at 42.0 and $31.3 \%$ of the initial amount after 32 days, respectively. The release kinetics were fitted well to Higuchi model, enabling the possibility of the prediction of release kinetics as a function of shell thickness thus achieving design-for-purpose silica nanocapsules. Furthermore, the nanocapsules showed excellent alkaline- and sunlight-shielding protective efficacies which resulted in significantly prolonged half-life of the sensitive cargo. Our biomimetic silica nanocapsules provide a nanocarrier platform for applications that demand process scalability, sustainability and biocompatibility coupled with unique cargo-protection and controlled-release properties. 


\section{INTRODUCTION}

In the past decades, a wide variety of nanomaterials has been developed as nanocarriers for encapsulation and release of various active ingredients for applications ranging from pharmaceutical and nutraceutical to agricultural and personal care. ${ }^{1-3}$ Ideally, these nanomaterials should facilitate high loading capacity with controllable release and protection as many actives (e.g., therapeutics, ${ }^{4}$ imaging agents, ${ }^{5}$ nutrients, ${ }^{6}$ agrochemicals, ${ }^{7}$ and fragrance ${ }^{8}$ ) are sensitive to local environments (e.g., heat, light, $\mathrm{pH}$ ) and hence prone to undergo degradation. However, it remains challenging to encapsulate and deliver these active agents while maintaining their stabilities, hence functionalities, for specific applications. A lot of efforts has been dedicated to develop new strategies for the design and synthesis of various types of nanomaterials based on polymer, ${ }^{9-11}$ lipid, ${ }^{12-14}$ and inorganic ${ }^{15-17}$ materials to protect and release such actives. Among them, inorganic silica nanomaterials have attracted significant interest due to their robust properties such as being mechanically strong, chemically stable, and relatively biocompatible. ${ }^{18-19}$

Inorganic silica nanocapsules are promising as nanocarriers owing to their unique core-shell architecture. ${ }^{20}$ The core retains the actives and provides high loading capacity, while the shell protects the actives from the surrounding environment and acts as a diffusion barrier that controls release of the encapsulated actives. Templating methods have been widely utilized to synthesize silica nanocapsules using either hard (i.e., solid nanoparticles ${ }^{21-22}$ ) or soft (i.e., emulsion droplets ${ }^{23-}$ 24 and gas bubbles ${ }^{25-26}$ ) templates. These approaches variously involve the use of chemical surfactants, organic solvents and template materials that are toxic, thus requiring tedious monitoring procedures for ensuring their complete removal down to the level that can be regarded as environmentally safe. Additionally, extreme $\mathrm{pH}$ and elevated temperature typically used during silica formation are obviously not suitable when sensitive active agents aforementioned are 
encapsulated prior to the silica formation. Although an active can be post-loaded into the prepared silica nanocapsules, these strategies are time consuming and often result in low loading capacity and initial burst release due to the adsorbed active present on the nanocapsules' surfaces. New methods for synthesizing silica nanocapsules for the encapsulation of sensitive materials using components and processes that are biocompatible and environmentally safe are urgently needed.

Inspired by Nature, biomimetic templating methods have emerged as relatively new approaches to synthesize silica-based nanocarriers owing to the abilities of mineralizing biomolecules to recognize, interact with, and direct the nucleation and growth of silica under physiological conditions. ${ }^{27-31}$ For example, Jakhmola et al. synthesized oil-core silica-shell nanocapsules thereby using a layer-by-layer assembly method in which oppositely charged biomolecules were alternately deposited on a nanoemulsion template followed by silica mineralization. ${ }^{32}$ Erni et al. reported the synthesis of silica capsules having a diameter at a scale of hundreds of micrometers by silicification of cross-linked coacervate of gelatin and Acacia gum around oil droplets. ${ }^{33}$

Recently, we developed a novel emulsion and biomimetic dual-templating technology to synthesize silica nanocapsules. ${ }^{24}$ This technology is based on the rational design of dual-functional peptides $^{24}$ or proteins $^{34}$ in lieu of chemical surfactants. The designed biomolecules are capable of stabilizing pharmaceutically-grade oil droplets (emulsification functionality) and concurrently inducing the formation silica shells (biomimetic-silicification functionality) at near-neutral $\mathrm{pH}$ and room temperature without the use of toxic organic solvents, ${ }^{24,}{ }^{34}$ thus eliminating adverse environmental effects. ${ }^{35-36}$ In addition, encapsulation of an active is facile through its direct dissolution in the oil core prior to the silica-shell formation, enabling high encapsulation efficiency and avoiding initial burst release. More importantly, the bifunctional proteins can be produced via a simple and cheap engineered bioprocess,${ }^{37}$ thus paving a pathway for large-scale economic 
production where cost is a competitive driver. While the bioenabled synthesis of silica nanocapsules has been described, release kinetics of an encapsulated active from silica nanocapsules as well as their capability of protecting the active under various conditions have not been studied

In this work, the performances of silica nanocapsules to encapsulate, protect and release a sensitive cargo were systematically investigated to better understand their stability, release mechanisms and kinetics under different conditions. Fipronil, that is sensitive to light and $\mathrm{pH}$, was used as a model active ingredient. Fipronil is an agrochemical that has been applied, for instance, in rice paddy fields ${ }^{38}$ which essentially expose it to both sunlight and soil acidity/alkalinity. Silica nanocapsules with two shell thicknesses were synthesized and used as nanocarriers for fipronil to study its encapsulation and sustained release, as well as its protective efficacies against simulated sunlight and different $\mathrm{pHs}$. This study will provide a rational basis for designing and synthesizing novel and rational nanocarriers with desirable properties for controlled release.

\section{EXPERIMENTAL SECTION}

Materials. Miglyol 812 (Caesar \& Loretz GmbH, Hilden, Germany) was purified using heattreated silica gel (Sigma-Aldrich Pty Ltd, Castle Hill, Australia) before use. The dual-functional protein D4S2 having an amino acid sequence of M(DPSMKQLADSLHQLARQVSRLEHA)4 EPSRKKRKKRKKRKKGGGY and a theoretical molecular weight $\left(\mathrm{M}_{\mathrm{W}}\right)$ of $13299.3 \mathrm{~g} / \mathrm{mol}$ was produced in our lab through recombinant gene expression using microbial cell factory ${ }^{34}$ and then purified by an engineered bioprocess. ${ }^{37}$ Fipronil $\left(\mathrm{C}_{12} \mathrm{H}_{4} \mathrm{C}_{12} \mathrm{~F}_{6} \mathrm{~N}_{4} \mathrm{OS}, \mathrm{M}_{\mathrm{W}} 437.15 \mathrm{~g} / \mathrm{mol}\right)$ was of analytical grade and was kindly provided by Accensi Pty Ltd (Narangba, Australia). Water having $>18.2 \mathrm{M} \Omega \mathrm{cm}$ resistivity was produced from a Milli-Q system with a $0.22 \mu \mathrm{m}$ filter (Merck 
Millipore, Bayswater, Australia). Other chemicals were of analytical grade obtained from either Merck or Sigma-Aldrich and used as received unless stated otherwise.

Synthesis and Characterization. Miglyol 812 oil containing fipronil $(10 \mathrm{~g} / \mathrm{L})$ was added to the D4S2 solution (1.53 g/L) in 4-(2-hydroxyethyl)-1-piperazineethanesulfonic acid (HEPES) buffer ( $25 \mathrm{mM}, \mathrm{pH} 7.5)$ at a volume ratio of 1 to 9 . Then the mixture was homogenized using an ultrasonicator (Branson Sonifier 450, Branson Ultrasonics, Danbury, CT) for four $30 \mathrm{~s}$ bursts at $40 \mathrm{~W}$ and interspersed in an ice bath for $60 \mathrm{~s}$ to form nanoemulsions. The nanoemulsions were diluted 5 times (the final concentration of fipronil is $200 \mu \mathrm{g} / \mathrm{mL}$ ) and then mixed with a silica precursor tetraethoxysilane (TEOS) at a concentration of 40 or $160 \mathrm{mM}$ under stirring at room temperature for $24 \mathrm{~h}$ to form fipronil-loaded silica nanocapsules having two different silica-shell thicknesses. The fipronil-loaded silica nanocapsules were collected by centrifugation $(10,000 \mathrm{~g}, 5$ min), and washed twice with ethanol and then twice with water.

Dynamic light scattering (DLS). Size distribution and zeta potential of nanoemulsions and silica nanocapsules were determined by DLS using Malvern Zetasizer Nano ZS (Malvern Instrument Ltd., Malvern, UK) at a scattering angle of $173^{\circ}$ and a temperature of $25^{\circ} \mathrm{C}$. The samples were diluted prior to the measurements to avoid multiple scattering effects.

Transmission electron microscopy (TEM). Morphology of silica nanocapsules was observed by TEM using a JEOL 1010 (JEOL, Tokyo, Japan) operated at $100 \mathrm{kV}$. The silica nanocapsule suspensions $(2 \mu \mathrm{L})$ were deposited onto Formvar-coated copper grids (ProSciTech, Townsville, Australia). The size and shell thickness of the silica nanocapsules were analyzed using iTEM software (Version 3.2, Soft Imaging System GmbH, Münster, Germany). 
Reversed-phase high-performance liquid chromatography (RP-HPLC). Concentrations of fipronil and D4S2 protein were analyzed using RP-HPLC equipped with a Jupiter C18 column (5 $\mu \mathrm{m} ; 300 \AA \AA ; 150 \mathrm{~mm} \times 4.6 \mathrm{~mm})($ Phenomenex, Torrance, $\mathrm{CA})$ and connected to an LC-10AVP series HPLC system. The mobile phase A was $0.1 \mathrm{v} / \mathrm{v} \%$ trifluoroacetic acid (TFA) in water, and the mobile phase $\mathrm{B}$ was $90 \mathrm{v} / \mathrm{v} \%$ acetonitrile, $0.1 \mathrm{v} / \mathrm{v} \%$ TFA in water. To measure the concentration of fipronil, a linear gradient from 50 to $70 \% \mathrm{~B}$ in $20 \mathrm{~min}$ at a flow rate of $1 \mathrm{~mL} / \mathrm{min}$ with a detection wavelength at $220 \mathrm{~nm}$ was used. Meanwhile, to determine the concentration of D4S2 protein, a linear gradient from 30 to $65 \% \mathrm{~B}$ in $35 \mathrm{~min}$ at $1-\mathrm{mL} / \mathrm{min}$ flowrate and $214-\mathrm{nm}$ wavelength was employed.

Encapsulation Efficiency. To measure the efficiency of fipronil encapsulation, acetonitrile was added to the suspensions of silica nanocapsules to completely extract fipronil from the oil as fipronil has high solubility in acetonitrile. ${ }^{24}$ Then the mixture was centrifuged to obtain the supernatant in which the concentration of fipronil was measured using RP-HPLC as described above. The encapsulation efficiency $(\% E E)$ was calculated as the ratio of the encapsulated amount of fipronil compared to the initial amount of fipronil dissolved in Miglyol 812 oil:

$$
\% E E=\left(1-\frac{C_{S} V_{s}}{C_{0} V_{0}}\right) \times 100 \%
$$

where $C_{s}(\mathrm{mg} / \mathrm{mL})$ and $V_{s}(\mathrm{mg} / \mathrm{mL})$ are the concentration of fipronil in the supernatant and the volume of the supernatant, respectively, and $C_{0}(\mathrm{mg} / \mathrm{mL})$ and $V_{0}(\mathrm{mg} / \mathrm{mL})$ are the initial concentration of fipronil in Miglyol 812 oil and the volume of Miglyol 812 oil, respectively.

In Vitro Release in Water. The release profiles of fipronil from nanoemulsions and silica nanocapsules having two different silica-shell thicknesses were investigated in water. The 
nanoemulsions and the silica nanocapsule suspensions (containing $200 \mu \mathrm{g}$ fipronil in $100 \mu \mathrm{L}$ ) were each sealed in dialysis tubing $(3.5 \mathrm{kDa})$ in $10 \mathrm{~mL}$ water and then shaken at room temperature for up to $32 \mathrm{~d}$. At different time intervals, aliquots $(200 \mu \mathrm{L})$ of the solution outside the dialysis tubing were taken and replaced with fresh water. Samples were then analyzed using RP-HPLC to determine the concentration of fipronil as described above.

In Vitro Release at different pHs. The release profiles of fipronil from nanoemulsions and silica nanocapsules having thickest shell thickness were studied in water and in solutions of $\mathrm{pH} 2$ and $\mathrm{pH}$ 11. Solutions of $\mathrm{pH} 2$ and $\mathrm{pH} 11$ were obtained by mixing $\mathrm{HCl}$ or $\mathrm{NaOH}$ with water, respectively. The nanoemulsions and the silica nanocapsule suspensions $(100 \mu \mathrm{L})$ were mixed with $10 \mathrm{~mL}$ water and $\mathrm{pH} 2-$ and $\mathrm{pH} 11$-solutions by shaking at room temperature for up to $32 \mathrm{~d}$. At different time intervals, aliquots $(500 \mu \mathrm{L})$ of the suspensions were taken and replaced with fresh water and $\mathrm{pH} 2$ - and $\mathrm{pH} 11$-solutions. All samples were neutralized to $\mathrm{pH} 7$ by adding $50 \mu \mathrm{L}$ of $\mathrm{NaOH}\left(\mathrm{pH}^{13}\right)$ or $\mathrm{HCl}\left(\mathrm{pH}_{2}\right)$ solution prior to fipronil analysis using RP-HPLC. For the neutralized silica nanocapsules, fipronil concentrations were examined both in the external solution and in the inside silica nanocapsules. To determine the fipronil concentration in the external solution, the silica nanocapsule suspension was centrifuged to obtain the supernatant which was then directly analyzed by using RP-HPLC. To determine the fipronil concentration inside the silica nanocapsules, the precipitates obtained after centrifugation were mixed with acetonitrile and then sonicated using ultrasonicator for eight $30 \mathrm{~s}$ bursts at $40 \mathrm{~W}$ and interspersed in an ice bath for $60 \mathrm{~s}$, followed by shaking for $48 \mathrm{~h}$ to completely extract fipronil to the bulk solutions. Then the mixtures were centrifuged to obtain the supernatants which were subjected to RP-HPLC analysis. After $32 \mathrm{~d}$, the silica nanocapsules with different $\mathrm{pH}$ treatment were visualized by using TEM. 
In Vitro Release under Simulated Sunlight. The release profiles of fipronil from the nanoemulsions and the silica nanocapsules having thickest shell thickness were investigated with two different treatments: under a solar simulator (67005 Arc Lamp Housing, Newport Corporation, Irvine, CA) at a light intensity of $1000 \mathrm{~W} / \mathrm{m}^{2}$ (equivalent to 1 sun) and under a dark condition by covering the samples with aluminium foil. The nanoemulsions and silica nanocapsules (100 $\mu \mathrm{L})$ were dispersed in $10 \mathrm{~mL}$ water under different radiation conditions at a room temperature for up to $72 \mathrm{~h}$. At different time intervals, aliquots $(500 \mu \mathrm{L})$ of the suspensions were taken, replaced with fresh water, and then centrifuged to obtain supernatants $(100 \mu \mathrm{L})$ which were subjected to fipronil analysis using RP-HPLC. On the other hand, the precipitates were resuspended and mixed with acetonitrile $(500 \mu \mathrm{L})$ then sonicated using a Branson Sonifier 450 ultrasonicator for eight $30 \mathrm{~s}$ bursts at $40 \mathrm{~W}$ and interspersed in an ice bath for $60 \mathrm{~s}$, followed by shaking for $48 \mathrm{~h}$. Then samples were centrifuged to obtain the supernatants which were analyzed by using RP-HPLC. After $72 \mathrm{~h}$, the silica nanocapsules with different radiation treatment were visualized by using TEM.

\section{RESULTS AND DISCUSSION}

Synthesis and Characterization of Silica Nanocapsules. Silica nanocapsules with tunable shell thicknesses were synthesized through an emulsion and biomimetic dual-templating technology using dual-functional protein D4S2 (Figure 1). The D4S2 protein was designed in our group by modularizing a surface-active module (M(DPSMKQLADS LHQLARQ VSRLEHA) 4 ) with a silica mineralization-active module (RKKRKKRKKRKKGGGY). Briefly, fipronil as a model sensitive cargo was first dissolved in a pharmaceutical-grade oil Miglyol 812 at a concentration of $10 \mathrm{~g} / \mathrm{L}$. The fipronil-loaded oil was emulsified in the presence of D4S2 protein by sonication. Stable oil-in-water nanoemulsions were thus formed because of the facial amphiphilicity of the $\alpha$-helical surface-active module of D4S2 protein that enabled the 
hydrophobic residues of the protein to face and anchor to the oil droplets, while their hydrophilic residues projected toward the aqueous phase providing steric and electrostatic stabilization. After adding the silica precursor tetraethoxysilane (TEOS) to the nanoemulsions, a silica shell encasing the nanoemulsion core was formed through a biosilicification process at $\mathrm{pH} 7.5$ and room temperature without using any organic solvents. The biosilicification-active module, which is densely packed with cationic amino acid residues as well as polar amino acid residues, oriented within the subinterfacial region between nanoemulsion and bulk solution induce the hydrolysis of TEOS and the polycondensation of the hydrolyzed TEOS at the oil-water interfaces through nucleophilic attack, H-bonding and electrostatic interaction mechanisms, ${ }^{24}$ hence ultimately forming silica shell encapsulating the oil core.

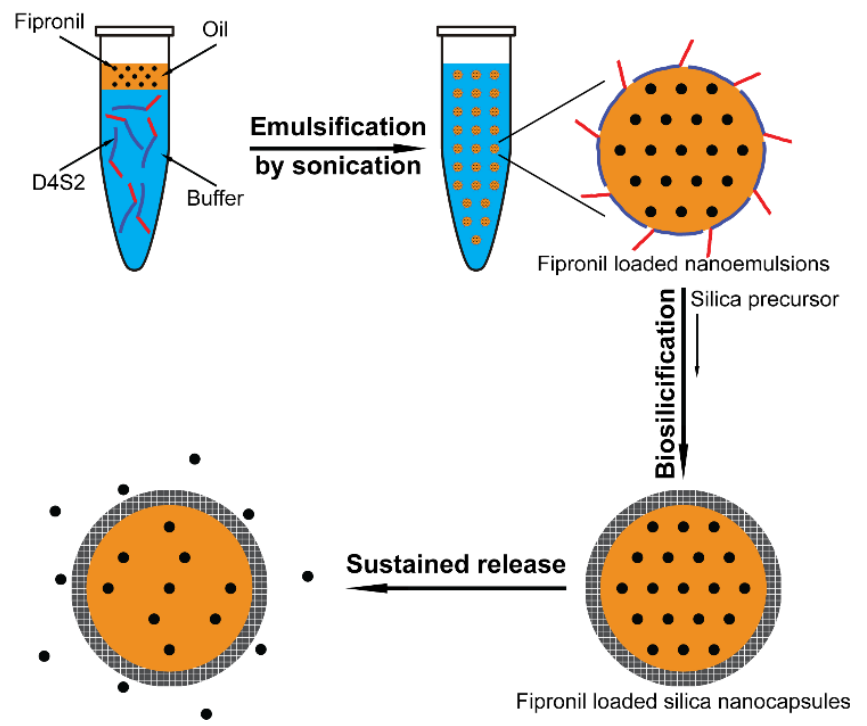

Figure 1. Schematic illustration of the emulsion and biomimetic dual-templating technology for the synthesis and use of biomimetic silica nanocapsules as nanocarriers. The fabrication process uses dual-functional protein D4S2 for: (i) facilitating the formation and stabilization of a nanoemulsion template, and (ii) catalyzing the nucleation and growth of silica at the oil-water 
interface under near-neutral $\mathrm{pH}$ and room temperature without the use of toxic organic solvents. The cargo (fipronil) is encapsulated by dissolving it in the oil prior to emulsification.

Figure 2A shows the size distribution of nanoemulsions (NEs) and silica nanocapsules having different shell thicknesses (SNCs-40 and SNCs-77) by using DLS. The hydrodynamic diameter of the nanoemulsions was $192 \pm 23 \mathrm{~nm}$ with a dispersity $(\nexists)$ of $0.18 \pm 0.02$ and a zeta potential of 59 $\pm 4 \mathrm{mV}$. The nanoemulsions were then used as templates for the synthesis of silica nanocapsules (SNCs-40 and SNCs-77) having a core-shell structure and different silica-shell thicknesses (Figure 2B,C). The two silica nanocapsules were synthesized by mixing the nanoemulsion templates with TEOS at concentrations of 40 and $160 \mathrm{mM}$ that resulted in uniform diameters of $270 \pm 19$ and 345 $\pm 13 \mathrm{~nm}$ with silica-shell thicknesses of $40 \pm 4$ and $77 \pm 2 \mathrm{~nm}$, respectively, as determined by using TEM. In contrast to the nanoemulsions which had a positive zeta potential because of the positively-charged $\mathrm{D} 4 \mathrm{~S} 2$ protein at $\mathrm{pH} 7.5$ present at the oil-water interface, the two silica nanocapsules having 40- and 77-nm shell thicknesses showed negative charges of $-13 \pm 2$ and $33 \pm 8 \mathrm{mV}$, respectively, as a result of the deprotonation of the silanol groups on the silica surface $\left(\equiv \mathrm{Si}-\mathrm{O}^{-}\right)$at $\mathrm{pH}$ 7.5. Furthermore, the encapsulation efficiencies of fipronil in the silica nanocapsules of 40 and $77 \mathrm{~nm}$-shells were $96.3 \pm 3.5$ and $97.5 \pm 2.1 \%$, respectively. Compared to other approaches for encapsulation, our nanocapsules demonstrated significantly higher encapsulation efficiencies..$^{21,39-40}$ 

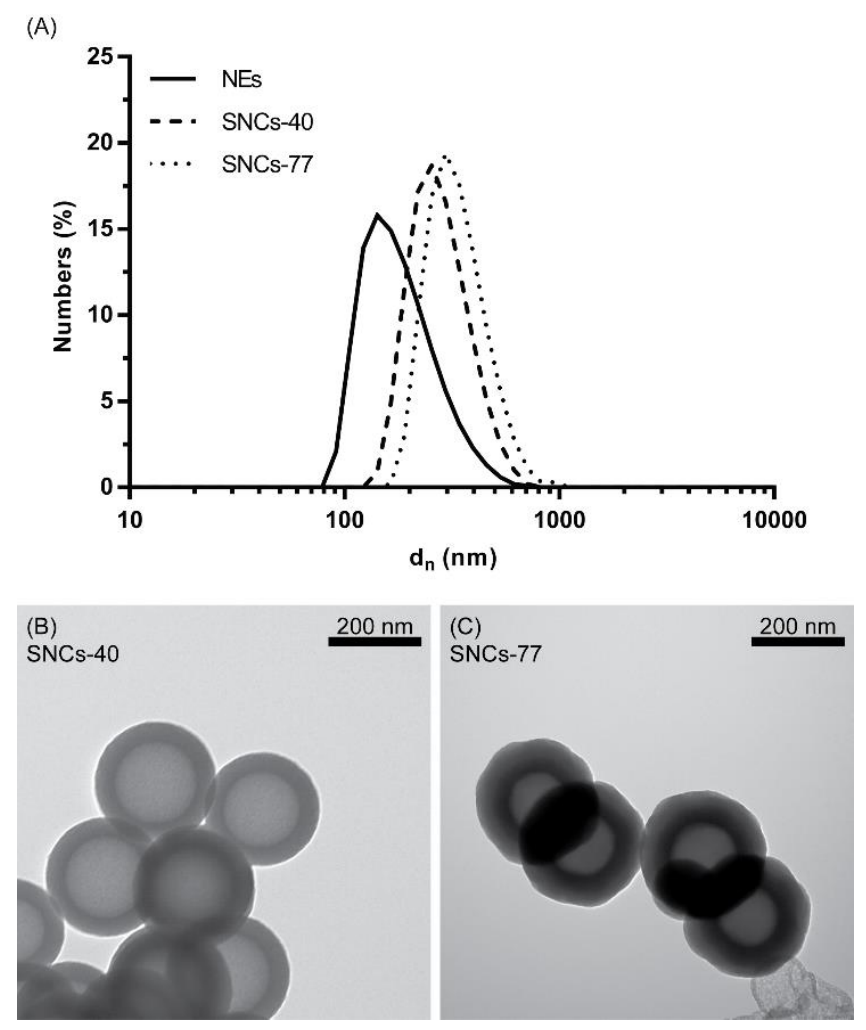

Figure 2. Characterization of fipronil-loaded nanoemulsions (NEs) and fipronil-loaded silica nanocapsules having shell thicknesses of 40 (SNCs-40) and 77 (SNCs-77) nm as determined by using dynamic light scattering (DLS) and transmission electron microscopy (TEM). (A) Size distributions obtained from DLS. (B, C) Morphology of silica nanocapsules having different silicashell thicknesses visualized under TEM.

Effect of Shell Thickness on the Fipronil Release. The effects of silica-shell thickness on the release kinetics of fipronil from nanoemulsions and silica nanocapsules were investigated in water for a period of $32 \mathrm{~d}$. Figure 3 shows the release profiles of fipronil from nanoemulsions having zero silica-shell thickness (NEs) and from silica nanocapsules having shell-thicknesses of 40 (SNCs-40) and 77 (SNCs-77) nm. Fipronil release from NEs showed a substantially rapid release compared to SNCs-40 and SNCs-77, and the release of fipronil from SNCs-77 was slower than that of SNCs-40. At $24 \mathrm{~h}$, only $11.5 \pm 1.2,7.6 \pm 1.7$ and $5.7 \pm 1.8 \%$ of the total encapsulated 
fipronil were released from NEs, SNCs-40 and SNCs-77, respectively. The release of fipronil then gradually increased with time. At $32 \mathrm{~d}, 64.6 \pm 4.2,42.0 \pm 3.8$ and $31.3 \pm 3.6 \%$ of the initially loaded fipronil were released from NEs, SNCs-40 and SNCs-77, respectively. The release rate of the three nanosystems in water is in the order of the NEs $>$ SNCs-44 $>$ SNCs-77, demonstrating that the silica shell was an effective diffusion barrier in slowing down the release of the encapsulated active, and the thicker the shell thickness the slower was the release. The silica nanocapsules demonstrated sustained release properties with no indication of initial burst release. This is in contrast with other silica nanoparticles which have significant amount of initial burst release. $^{41-42}$

The release kinetics of fipronil were fitted to different models. It was found that the release of fipronil from NEs, SNCs-40 and SNCs-77 can be well described by the Higuchi model (Figure 3):

$$
Q=k_{H} \cdot t^{1 / 2}
$$

where $Q$ is the accumulated release of fipronil (\%), $k_{H}$ is the Higuchi constant, and $t^{1 / 2}$ is the square root of time. Good linear correlations (regression from the Higuchi model; $R^{2}>0.95$ ) can be observed (Figure 3), suggesting that the release of our silica nanocapsules are controlled by diffusion. Based on the Higuchi model, the complete release of fipronil can be achieved at 69, 158 and $286 \mathrm{~d}$ for the NEs, SNCs-40 and SNCs-77, respectively, demonstrating a significant role of the silica shell in slowing down the release consistent with the experimental results described above. We have observed a linear relationship between the shell thickness and the Higuchi constant based on the three shell thicknesses $(0,40$, and $77 \mathrm{~nm})$ (Figure $\mathrm{S} 1$ ), which may enable us to predict the release profiles based on the shell thickness thus achieving purposely designed silica 
nanocapsules for controlled release. Further experiments will be conducted to confirm the accuracy of the correlation.

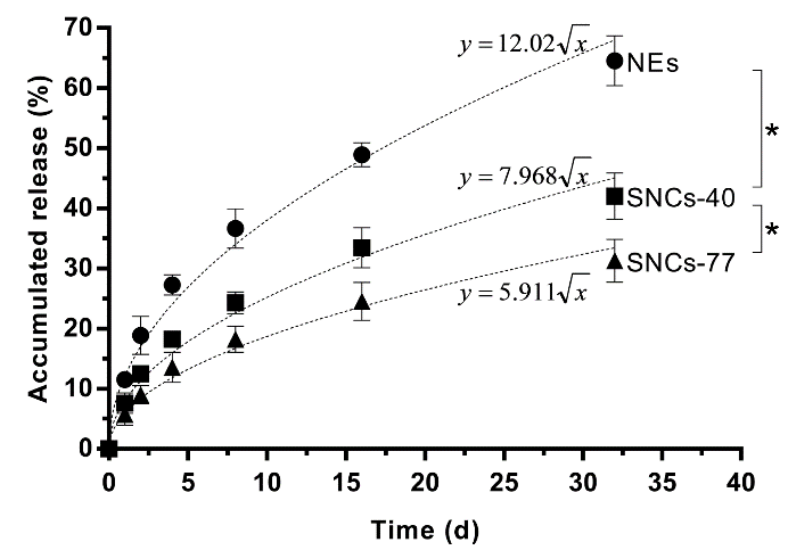

Figure 3. Release profiles of fipronil from nanoemulsions (NEs) and silica nanocapsules having 40 (SNCs-40) and 77 (SNCs-77) nm shell thicknesses in water. The dot points and the dotted lines represent the experimental data and the nonlinear regression to the Higuchi model, respectively. One-way ANOVA was used to determine statistical significance, with $\mathrm{P}$ value $<0.05$ denoted as *.Effect of $\mathbf{p H}$ on the Fipronil Release. The effects of $\mathrm{pH}$ on the release profiles of fipronil from the silica nanocapsules having thickest shell thickness (SNCs-77) were investigated in a 32-d period. Figure 4 shows the release of fipronil from SNCs-77 in water and in solutions of $\mathrm{pH} 2$ and $\mathrm{pH} 11$. As fipronil can be rapidly hydrolyzed at $\mathrm{pH} 11$, the concentration of fipronil inside the nanocapsules was measured by first disrupting the core-shell structure of nanocapsules and then fipronil released out of the nanocapsules can be neutralized prior to analysis using RP-HPLC. The SNCs-77 demonstrated a sustained release of fipronil at all $\mathrm{pH}$ conditions. The fipronil release was much faster at $\mathrm{pH} 11$ than those at $\mathrm{pH} 2$ and in water, indicating that the silica shell was less stable at $\mathrm{pH} 11$. At the end of the 32-d period, the accumulated fipronil release in water and in solutions of $\mathrm{pH} 2$ and $\mathrm{pH} 11$ were $35.7 \pm 3.9,56.1 \pm 2.5$ and $84.8 \pm 2.9 \%$, respectively. After 32 -d release 
experiments, the silica nanocapsules were observed by using TEM (Figure 5). The silica nanocapsules incubated at $\mathrm{pH} 2$ showed a unique hedgehog-like morphology (Figure 5A), and the shell thickness decreased significantly from $77 \pm 2$ to $26 \pm 9 \mathrm{~nm}$, indicating the gradual degradation of the silica shell. The silica nanocapsules incubated in water remained slightly intact with a rough topology on their surfaces (Figure 5B), and the shell thickness decreased slightly from $77 \pm 2$ to $67 \pm 6 \mathrm{~nm}$. At pH 11, most silica nanocapsules disintegrated into small silica nanoparticles with a diameter ranging from 1 to $20 \mathrm{~nm}$ (Figure 5C). All silica nanocapsules incubated at the conditions aforementioned demonstrated degradability dependent on solution $\mathrm{pH}$. This is because amorphous silica can undergo hydrolysis to soluble silanols $(\equiv \mathrm{Si}-\mathrm{OH})$ by breaking the siloxane bonds $(\equiv \mathrm{Si}-$ $\mathrm{O}-\mathrm{Si} \equiv)$ which is further catalyzed by either acid or alkaline. ${ }^{43}$ Moreover, the TEM images of the silica nanocapsules (Figure 5) corresponded well with the release profiles at three different $\mathrm{pHs}$ (Figure 4), as the more stable the shell, the slower was the release.

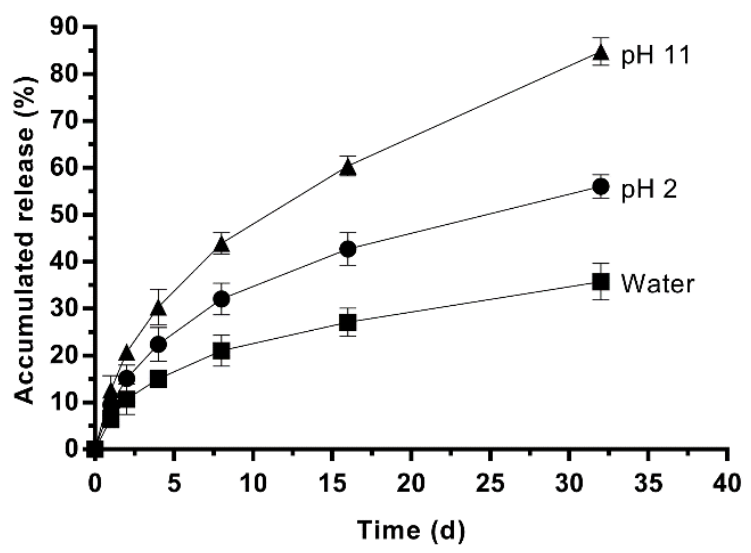

Figure 4. Release profiles of fipronil from the silica nanocapsules having $77 \mathrm{~nm}$-shell in water and in solutions of $\mathrm{pH} 2$ and $\mathrm{pH} 11$. 

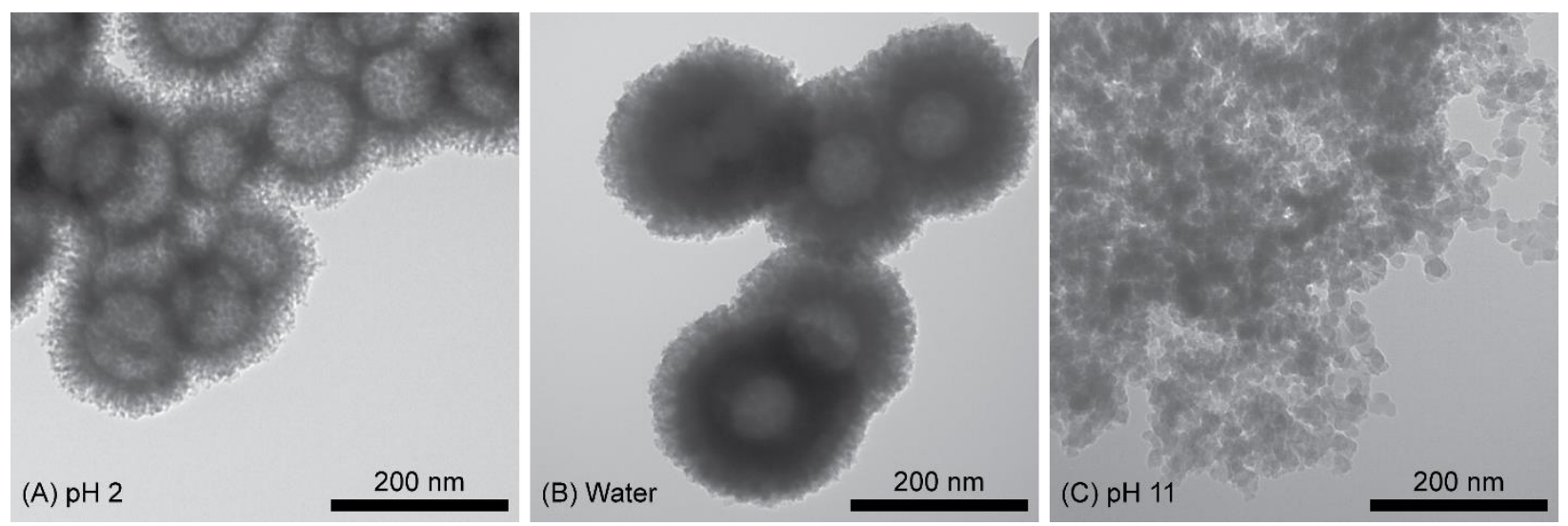

Figure 5. Surface morphology of silica nanocapsules having $77 \mathrm{~nm}$-shell after 32 days of incubation under different conditions: (A) $\mathrm{HCl}$ solution of $\mathrm{pH}$ 2; (B) Water; and (C) $\mathrm{NaOH}$ solution of pH 11.

Protective Efficacy of Silica Shell against Alkaline Conditions. An effective protection for the cargoes encapsulated in nanocarriers is essential as many of the cargoes (e.g., drugs, agrochemicals, imaging agents, proteins, and DNAs) are highly sensitive to acidic and/or basic conditions during various applications, resulting in degradation hence loss of functionalities. Fipronil herein was used as a model sensitive active ingredient, which undergo rapid hydrolysis under alkaline conditions to 5-amino-3-carbamoyl-1-(2,6-dichloro-4-(trifluoromethyl)-phenyl)-4[(trifluoromethyl)sulfinyl]pyrazole. ${ }^{44}$ Therefore, the protective efficacy of the silica shell for the encapsulated fipronil against degradation was investigated. Silica nanocapsules having $77 \mathrm{~nm}$ shell thickness (SNCs-77) were used in comparison with the nanoemulsions (NEs) at three different conditions i.e., water and solutions of $\mathrm{pH} 2$ and $\mathrm{pH} 11$. Figure 6 shows the variation of $C_{t} / C_{t 0}$ (i.e., the ratio of the total concentration of fipronil inside and outside (the amount that was released out) of either nanoemulsions or nanocapsules to its initial concentration) over $32 \mathrm{~d}$. The $C_{t} / C_{0}$ (Figure 6) represents the degradation rate of fipronil. The total amount of fipronil for both the nanoemulsions and the silica nanocapsules remained unchanged in $\mathrm{HCl}$ solution of $\mathrm{pH} 2$ (NEs-pH 
2 and SNCs-77-pH 2) and in water (NEs-Water and SNCs-77-Water), indicating its stability under these conditions. However, for the nanoemulsions incubated at $\mathrm{pH} 11$ (NEs-pH 11), the $C_{t} / C_{t 0}$ decreased significantly from $100 \%$ to $80.2 \pm 3.4 \%$ in only $3 \mathrm{~h}$, and then remained declining until no fipronil could be detected at day 4 , indicating a complete hydrolysis of fipronil. The $C_{t} / C_{0}$ vs time follows a pseudo-first-order reaction (half-life $=0.83 \mathrm{~d}$ ):

$$
r=k \cdot C_{t} \cdot C_{O H}
$$

where $r$ is the hydrolysis rate, $k$ is the second-order constant, $C_{t}$ is the total concentration of the remaining fipronil in the system, and $\mathrm{COH}^{-}$is the concentration of hydroxide ion. The $\mathrm{COH}^{-}$is considered as a constant in this study, therefore the hydrolysis rate becomes:

$$
r=k^{\prime} \cdot C_{t}
$$

where $k^{\prime}$ is the pseudo-first order constant, making the hydrolysis process a pseudo-first-order reaction. In comparison, fipronil in the silica nanocapsules with a shell thickness of $77 \mathrm{~nm}$ at $\mathrm{pH}$ 11 (SNCs-77-pH 11) demonstrated a much slower degradation rate (Figure 6). At $32 \mathrm{~d}$ there was still $15.3 \pm 2.9 \%$, revealing that the half-life of fipronil encapsulated in the silica nanocapsules at pH 11 had been prolonged by approximately 11 times, i.e., $0.83 \mathrm{~d}$ for the nanoemulsions (NEs-pH 11) and $8.93 \mathrm{~d}$ for the silica nanocapsules (SNCs-77-pH 11). These results demonstrated a remarkable protective efficacies of the silica shell for fipronil against degradation under alkaline conditions in comparison to the nanoemulsions which do not have silica shell. In contrast to other materials which rely on adsorption of cargoes on the surface or porous structure, ${ }^{21,39,41}$ our silica nanocapsules can retain most cargo in the oil core, as indicated by the absence of initial burst release (Figures 3 and 5), thus prolonging the lifetime of those active ingredients which are prone to undergo degradation. 


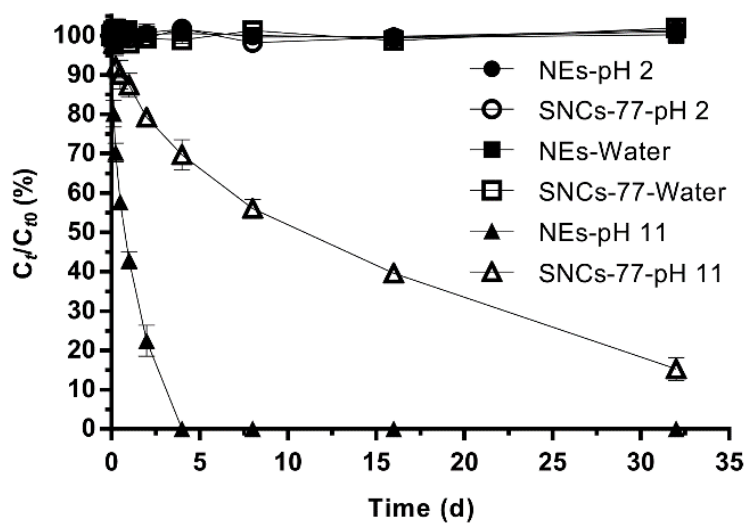

Figure 6. Effect of pHs on the degradation kinetics of fipronil encapsulated in nanoemulsions and silica nanocapsules having $77 \mathrm{~nm}$-shell. Variation of the total fipronil amount remained in the nanoemulsion and silica nanocapsules in water (NEs-Water and SNCs-77-Water) and in solutions of pH 2 (NEs-pH 2 and SNCs-77-pH 2) and pH 11 (NEs-pH 11 and SNCs-77-pH 11) are presented.

Protective Efficacy of Silica Shell against Sunlight. Driven by the fact that many active ingredients, such as ibuprofen, ${ }^{45}$ darbepoetin- $\alpha,{ }^{46}$ vinclozolin, ${ }^{47}$ and hydramethylnon, ${ }^{48}$ are light sensitive and thus require a great care during long-term storage, we also investigated the efficacies of silica nanocapsules for protection of encapsulated actives against light. Fipronil encapsulated in silica nanocapsules having $77 \mathrm{~nm}$-shell was used in this study as it is also sensitive to light hence prone to undergo photolysis. ${ }^{44}$ A solar simulator $\left(1000 \mathrm{~W} / \mathrm{m}^{2}\right.$, equivalent to 1 sun intensity) was used to mimic natural sunlight. Figure 7 shows the variation of $C_{t} / C_{0}$ (i.e., the ratio of the total concentration of fipronil inside and outside the nanoemulsions or nanocapsules to its initial concentration) under light exposure and dark condition over a 72 -h period. The $C_{t} / C_{0}$ (Figure 7) represents the degradation rate of fipronil under the light. The total concentrations of fipronil remained unchanged under dark condition for both the nanoemulsions (NEs-D) and silica nanocapsules (SNCs-77-D). However, the concentrations of fipronil in the nanoemulsions under 
light (NEs-L) decreased dramatically to $68.9 \pm 4.1 \%$ at $1.13 \mathrm{~h}$, and it was completely degraded at $9 \mathrm{~h}$. The degradation of fipronil followed a first-order reaction model with a degradation half-life of $2.27 \mathrm{~h}$. In contrast, the concentrations of fipronil in the silica nanocapsules under light exposure (SNCs-77-L) demonstrated a much slower degradation with $95.4 \pm 3.2$ and $83.9 \pm 0.3 \%$ fipronil remaining at 1.13 and $9 \mathrm{~h}$, respectively. At the end of the $72 \mathrm{~h}$ light-exposure experiment, $61.5 \pm$ $1.0 \%$ of the total fipronil was still present. The results demonstrated that the silica shell provided an excellent sunlight-shielding ability and thus protected the light-sensitive fipronil from photodegradation.

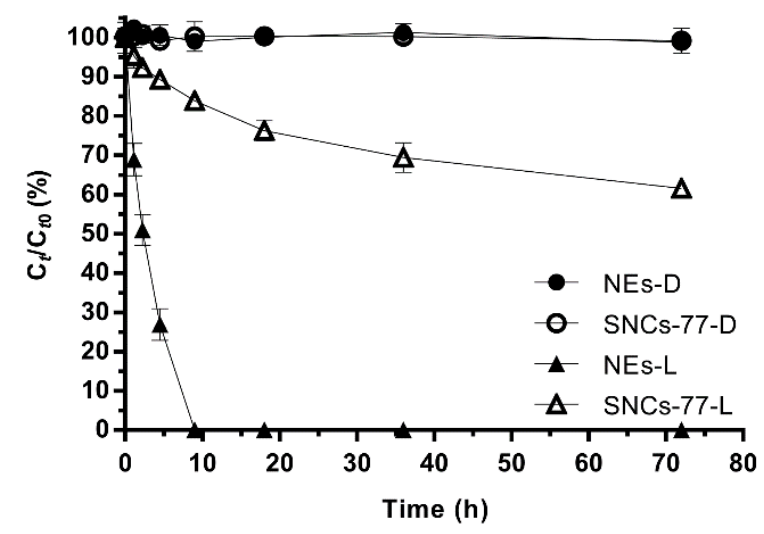

Figure 7. Effect of light exposure $\left(1000 \mathrm{~W} / \mathrm{m}^{2}\right)$ on the degradation kinetics of fipronil encapsulated in nanoemulsions and silica nanocapsules having $77 \mathrm{~nm}$-shell. Variation of the total fipronil amount remained in the nanoemulsions and silica nanocapsules over $72 \mathrm{~h}$ of incubation under light irradiation (NEs-L and SNCs-77-L) and dark condition (NEs-D and SNCs-77-D) are presented.

The concentrations of fipronil inside the silica nanocapsules were calculated and compared under light exposure and dark condition to determine whether fipronil inside the nanocapsules remained intact. Figure $8 \mathrm{~A}$ shows the variation of $C_{i} / C_{0}$ (i.e., the ratio of the fipronil concentration inside the silica nanocapsules to its initial concentration) under simulated light and dark conditions 
over a 72-h period. The silica nanocapsules under dark condition (In-SNCs-77-D) showed a sustained release of fipronil with $87.1 \pm 1.4 \%$ of the fipronil remained inside the nanocapsules at $72 \mathrm{~h}$. In contrast, the concentration of fipronil inside the silica nanocapsules under light (In-SNCs77-L) decreased more rapidly i.e., $61.5 \pm 1.0 \%$ at $72 \mathrm{~h}$. This result indicated that the silica shell provided certain level of protection against light degradation, as silica is optically transparent, ${ }^{49}$ and the protection efficacy was around $70.6 \%(=61.5 / 87.1)$. Figure $8 \mathrm{~B}$ shows the variation of the $C_{e} / C_{0}$ (i.e., the ratio of the fipronil concentration in the external buffer solution to the initial concentration) under both dark and light conditions over a 72-h period. It is clear that fipronil was released gradually from the silica nanocapsules that incubated under dark condition (Ex-SNCs-77D). As expected, no fipronil can be detected for those silica nanocapsules incubated under light condition (Ex-SNCs-77-L). This is because once fipronil released from the silica nanocapsules it exposed to the light and underwent rapid photo-degradation. 

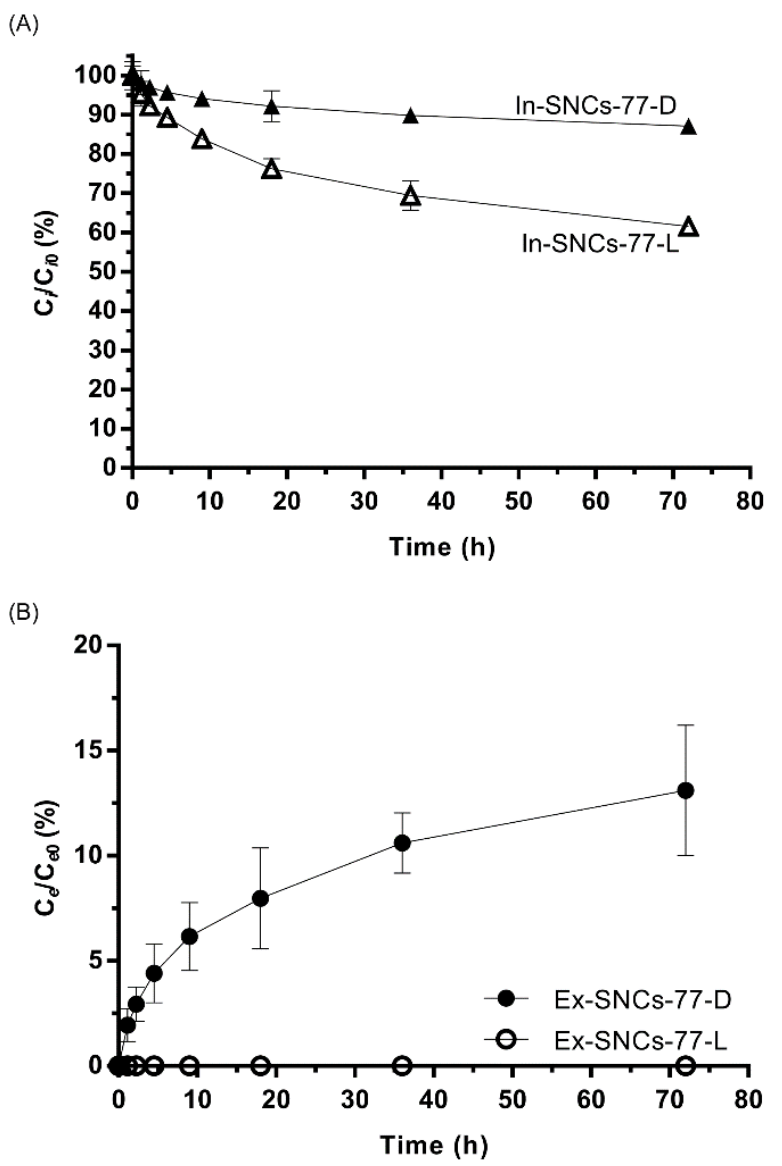

Figure 8. Efficacies of silica nanocapsules for the protection of the encapsulated fipronil against light exposure $\left(1000 \mathrm{~W} / \mathrm{m}^{2}\right)$. (A) Variation of the fipronil concentrations in the dispersed phase (inside the silica shell) of the silica nanocapsules having $77 \mathrm{~nm}$-shell with (In-SNCs-77-L) and without (In-SNCs-77-D) light irradiation. (B) Variation of the fipronil concentrations in the continuous phase (outside the silica shell) of the silica nanocapsules having $77 \mathrm{~nm}$-shell with (ExSNCs-77-L) and without (Ex-SNCs-77-D) light irradiation.

The physicochemical properties (size, size distribution, zeta potential and surface morphology) of nanoemulsions and silica nanocapsules having $77 \mathrm{~nm}$-shell after $72 \mathrm{~h}$-incubation under light $\left(1000 \mathrm{~W} / \mathrm{m}^{2}\right)$ were further examined. Figure 9A shows the DLS results of nanoemulsions and silica nanocapsules before and after exposure to light. The nanoemulsions exhibited a significant size 
increase from $192 \pm 23 \mathrm{~nm}$ before the irradiation (NEs-BF-L) to $510 \pm 43 \mathrm{~nm}$ after the irradiation (NEs-AT-L), whereas no difference was observed for the silica nanocapsules before (SNCs-77BF-L) and after (SNCs-77-AT-L) the irradiation (Figure 9A). The $Ð$ of the nanoemulsions also experienced a sharp increase (from $0.18 \pm 0.02$ to $0.63 \pm 0.04$ ) after the irradiation. This result indicate that the protein covering the nanoemulsion may undergo structural changes under light exposure $^{50}$ which caused irreversible aggregation of the nanoemulsions. In contrast, the $Ð$ of the silica nanocapsules remained relatively unchanged. The zeta potential of the nanoemulsions changed from $59 \pm 4$ to $-12 \pm 5 \mathrm{mV}$ after irradiation, while the silica nanocapsules showed a stable zeta potential of $-33 \pm 8 \mathrm{mV}$ during the $72 \mathrm{~h}$-incubation. Furthermore, the TEM images show intact core-shell structure of the silica nanocapsules with good dispersity after $72 \mathrm{~h}$ of light exposure (Figure 9B), consistent with the DLS results (Figure 9A). These results demonstrated the excellent stability of the silica nanocapsules against sunlight irradiation, unlike nanoemulsions.

(A)

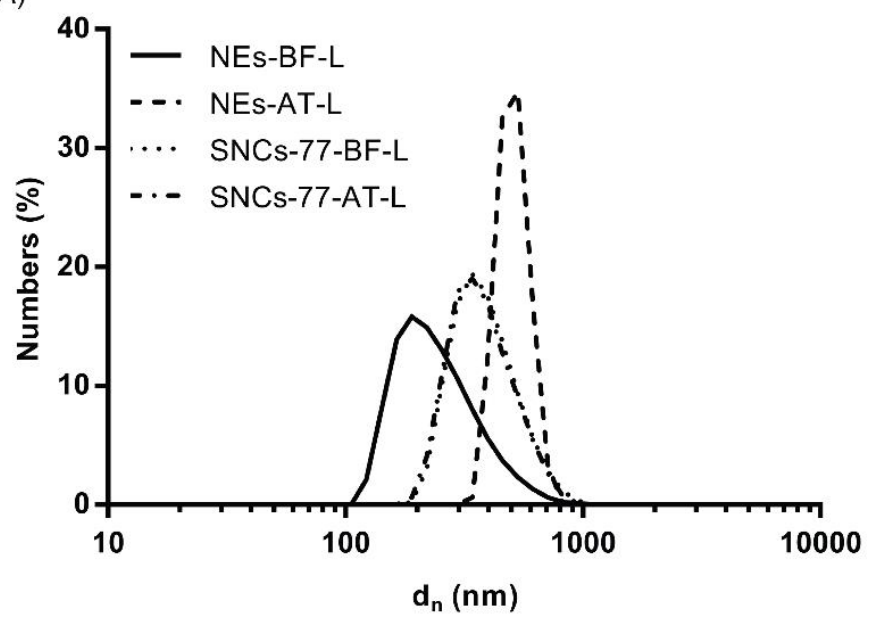

(B)

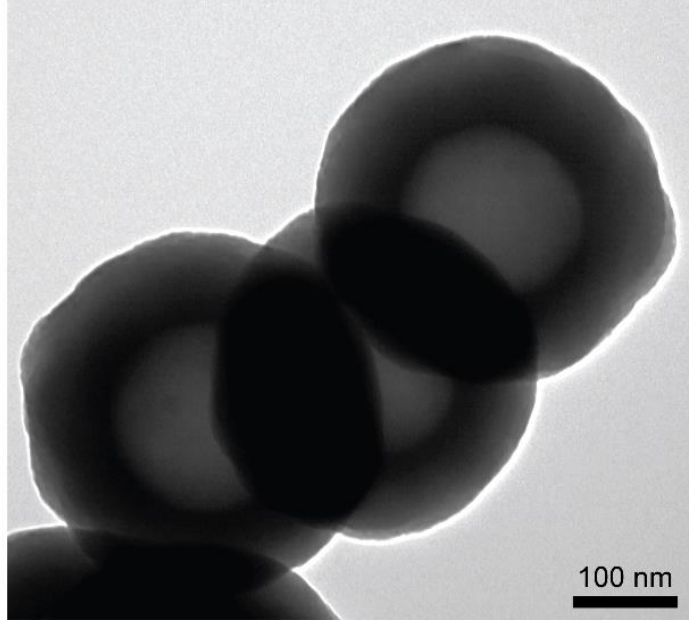

Figure 9. Effect of light exposure $\left(1000 \mathrm{~W} / \mathrm{m}^{2}, 72 \mathrm{~h}\right)$ to the physicochemical properties of nanoemulsions and silica nanocapsules having $77 \mathrm{~nm}$-shell as determined by using dynamic light scattering (DLS) and transmission electron microscopy (TEM). (A) Size distribution of 
nanoemulsions and silica nanocapsules before (NEs-BF-L and SNCs-77-BF-L) and after (NEsAT-L and SNCs-77-AT-L) light irradiation. (B) Structure of silica nanocapsules after light irradiation (SNCs-77-AT-L).

\section{CONCLUSIONS}

Biocompatible silica nanocapsules having an oil-core silica shell structure with different shell thicknesses of 40 and $77 \mathrm{~nm}$ were synthesized using an emulsion-biosilicification dual-templating technology, and high encapsulation efficiencies (96.3 and 97.5\%) were achieved. The release studies demonstrated an inverse relationship between the shell thickness and the release rate, and the release profiles fitted well to the Higuchi model. The silica nanocapsules showed different release kinetics under three conditions (water and solutions of $\mathrm{pH} 2$ and $\mathrm{pH} 11$ ) with accumulated release rates of $35.7,56.1$ and $84.8 \%$, respectively, after $32 \mathrm{~d}$ of incubation. In addition to the fipronil release, the $\mathrm{pH}$ of the solutions where nanocapsules were dispersed influenced the degradation of silica. The silica shell degraded slightly in water but experienced significant changes at $\mathrm{pH} 2$ and $\mathrm{pH}$ 11. Especially for $\mathrm{pH}$ 11, the silica nanocapsules disintegrated into small nanoparticles after $32 \mathrm{~d}$. However, compared to the nanoemulsions, the silica nanocapsules showed excellent protection of the encapsulated fipronil against degradation at $\mathrm{pH} 11$ as the half-life of fipronil within the nanocapsules was prolonged by approximately 11 times. Furthermore, silica nanocapsules demonstrated a $70.6 \%$ protection efficiency for the encapsulated fipronil after $72-\mathrm{h}$ irradiation to simulated sunlight $\left(1000 \mathrm{~W} / \mathrm{m}^{2}\right)$. In contrast, the fipronil loaded in the nanoemulsions experienced complete degradation within $9 \mathrm{~h}$. This work systematically investigated the release properties of our silica nanocapsules under different $\mathrm{pH}$ and light conditions, providing a fundamental understanding of this unique nanosystem for potential applications in controlled release. 


\section{AUTHOR INFORMATION}

\section{Corresponding Author}

*Phone: +61-7-3346-4263; e-mail: z.chunxia@uq.edu.au (C.-X.Z.).

\section{Author Contributions}

The manuscript was written through the contributions of all authors. All authors have given approval to the final version of the manuscript.

\section{Notes}

The University of Queensland (UQ) filed patent on the use of mineralizing proteins for making silica nanocapsules. A.P.J.M., C.-X.Z., and D.W. are named inventors on this patent and through their employment with UQ hold an indirect interest in this intellectual property. The other authors declare no conflict of interest.

\section{ACKNOWLEDGMENTS}

This work was supported by the Australian Research Council (ARC) under Discovery Project (DP150100798) and a Future Fellowship Project (FT140100726). C.-X.Z. acknowledges financial support from the award of the ARC Future Fellowship (FT140100726). National Collaborative Research Infrastructure Strategy (NCRIS) is acknowledged for support with the reversed-phase high-performance liquid chromatography. The authors also acknowledge the facilities and the scientific and technical assistance of the Australian Microscopy \& Microanalysis Research Facility at the Centre for Microscopy and Microanalysis, The University of Queensland.

\section{ABBREVIATIONS}


A, alanine; D, aspartic acid; DLS, dynamic light scattering; E, glutamic acid; EE, encapsulation efficiency; G, glycine; H, histidine; HEPES, 2-[4-(2-hydroxyethyl)piperazin-1-yl]ethanesulfonic acid; K, lysine; L, leucine; $\mathrm{M}$, methionine; $\mathrm{M}_{\mathrm{w}}$, molecular weight; $\mathrm{NEs}$, nanoemulsions; $\mathrm{P}$, proline; Q, glutamine; R, arginine; RP-HPLC, reversed-phase high-performance liquid chromatography; S, serine; SNCs, silica nanocapsules; TEM, transmission electron microscopy; TEOS, tetraethoxysilane; TFA, trifluoroacetic acid; $\mathrm{V}$, valine; $\mathrm{Y}$, tyrosine; $\oslash$, dispersity.

\section{REFERENCES}

(1) Farokhzad, O. C.; Langer, R. Impact of Nanotechnology on Drug Delivery. ACS Nano 2009, 3 (1), 16-20.

(2) Dasgupta, N.; Ranjan, S.; Mundekkad, D.; Ramalingam, C.; Shanker, R.; Kumar, A. Nanotechnology in Agro-Food: from Field to Plate. Food Res. Int. 2015, 69, 381-400.

(3) Kaur, I. P.; Agrawal, R. Nanotechnology: a New Paradigm in Cosmeceuticals. Recent Pat. Drug Delivery Formulation 2007, 1 (2), 171-182.

(4) Nielsen, P. E.; LEICK, V.; Buchardt, O. Light Sensitive Chloramphenicol Analogues. Acta Chem. Scand., Ser. B 1975, 29, 662-66.

(5) Eggeling, C.; Widengren, J.; Rigler, R.; Seidel, C. Photobleaching of Fluorescent Dyes under Conditions Used for Single-Molecule Detection: Evidence of Two-Step Photolysis. Anal. Chem. 1998, 70 (13), 2651-2659.

(6) Vikram, V.; Ramesh, M.; Prapulla, S. Thermal Degradation Kinetics of Nutrients in Orange Juice Heated by Electromagnetic and Conventional Methods. J. Food Eng. 2005, 69 (1), $31-40$.

(7) Katsumata, H.; Okada, T.; Kaneco, S.; Suzuki, T.; Ohta, K. Degradation of Fenitrothion by Ultrasound/Ferrioxalate/UV System. Ultrason. Sonochem. 2010, 17 (1), 200-206.

(8) Sköld, M.; Karlberg, A.-T.; Matura, M.; Börje, A. The Fragrance Chemical $\beta$ Caryophyllene-Air Oxidation and Skin Sensitization. Food Chem. Toxicol. 2006, 44 (4), 538545.

(9) Gref, R.; Minamitake, Y.; Peracchia, M. T.; Trubetskoy, V.; Torchilin, V.; Langer, R. Biodegradable Long-Circulating Polymeric Nanospheres. Science 1994, 263 (5153), 1600-1603.

(10) Stuart, M. A. C.; Huck, W. T.; Genzer, J.; Müller, M.; Ober, C.; Stamm, M.; Sukhorukov, G. B.; Szleifer, I.; Tsukruk, V. V.; Urban, M. Emerging Applications of Stimuli-Responsive Polymer Materials. Nat. Mater. 2010, 9 (2), 101-113.

(11) Soppimath, K. S.; Aminabhavi, T. M.; Kulkarni, A. R.; Rudzinski, W. E. Biodegradable Polymeric Nanoparticles as Drug Delivery Devices. J. Controlled Release 2001, 70 (1), 1-20.

(12) MuĖller, R. H.; MaĖder, K.; Gohla, S. Solid Lipid Nanoparticles (SLN) for Controlled Drug Delivery-a Review of the State of the Art. Eur. J. Pharm. Biopharm. 2000, 50 (1), 161-177.

(13) Mehnert, W.; Mäder, K. Solid Lipid Nanoparticles: Production, Characterization and Applications. Adv. Drug Delivery Rev. 2001, 47 (2), 165-196. 
(14) Wissing, S.; Kayser, O.; Müller, R. Solid Lipid Nanoparticles for Parenteral Drug Delivery. Adv. Drug Delivery Rev. 2004, 56 (9), 1257-1272.

(15) Cushing, B. L.; Kolesnichenko, V. L.; O'Connor, C. J. Recent Advances in the LiquidPhase Syntheses of Inorganic Nanoparticles. Chem. Rev. 2004, 104 (9), 3893-3946.

(16) Daniel, M.-C.; Astruc, D. Gold Nanoparticles: Assembly, Supramolecular Chemistry, Quantum-Size-Related Properties, and Applications toward Biology, Catalysis, and Nanotechnology. Chem. Rev. 2004, 104 (1), 293-346.

(17) Lai, C.-Y.; Trewyn, B. G.; Jeftinija, D. M.; Jeftinija, K.; Xu, S.; Jeftinija, S.; Lin, V. S.-Y. A Mesoporous Silica Nanosphere-Based Carrier System with Chemically Removable CdS Nanoparticle Caps for Stimuli-Responsive Controlled Release of Neurotransmitters and Drug Molecules. J. Am. Chem. Soc. 2003, 125 (15), 4451-4459.

(18) Torney, F.; Trewyn, B. G.; Lin, V. S.-Y.; Wang, K. Mesoporous Silica Nanoparticles Deliver DNA and Chemicals into Plants. Nat. Nanotechnol. 2007, 2 (5), 295-300.

(19) Lou, X. W. D.; Archer, L. A.; Yang, Z. Hollow Micro - /Nanostructures: Synthesis and Applications. Adv. Mater. 2008, 20 (21), 3987-4019.

(20) Wibowo, D.; Hui, Y.; Middelberg, A. P.; Zhao, C.-X. Interfacial Engineering for Silica Nanocapsules. Adv. Colloid Interface Sci. 2016.

(21) Chen, J.-F.; Ding, H.-M.; Wang, J.-X.; Shao, L. Preparation and Characterization of Porous Hollow Silica Nanoparticles for Drug Delivery Application. Biomaterials 2004, 25 (4), 723-727.

(22) Wang, Y.; Wang, K.; Zhang, R.; Liu, X.; Yan, X.; Wang, J.; Wagner, E.; Huang, R. Synthesis of Core-Shell Graphitic Carbon@ Silica Nanospheres with Dual-Ordered Mesopores for Cancer-Targeted Photothermochemotherapy. ACS Nano 2014, 8 (8), 7870-7879.

(23) Chang, F.-P.; Hung, Y.; Chang, J.-H.; Lin, C.-H.; Mou, C.-Y. Enzyme Encapsulated Hollow Silica Nanospheres for Intracellular Biocatalysis. ACS Appl. Mater. Interfaces 2014, 6 (9), 6883-6890.

(24) Wibowo, D.; Zhao, C.-X.; Middelberg, A. P. Emulsion-Templated Silica Nanocapsules Formed Using Bio-Inspired Silicification. Chem. Commun. 2014, 50 (77), 11325-11328.

(25) Rana, R. K.; Mastai, Y.; Gedanken, A. Acoustic Cavitation Leading to the Morphosynthesis of Mesoporous Silica Vesicles. Adv. Mater. 2002, 14 (19), 1414-1418.

(26) Wang, J.-G.; Li, F.; Zhou, H.-J.; Sun, P.-C.; Ding, D.-T.; Chen, T.-H. Silica Hollow Spheres with Ordered and Radially Oriented Amino-Functionalized Mesochannels. Chem. Mater. 2009, 21 (4), 612-620.

(27) Kröger, N.; Deutzmann, R.; Sumper, M. Polycationic peptides from diatom biosilica that direct silica nanosphere formation. Science 1999, 286 (5442), 1129-1132.

(28) Luckarift, H. R.; Spain, J. C.; Naik, R. R.; Stone, M. O. Enzyme immobilization in a biomimetic silica support. Nat. Biotechnol. 2004, 22 (2), 211-213.

(29) Yokoi, T.; Sakamoto, Y.; Terasaki, O.; Kubota, Y.; Okubo, T.; Tatsumi, T. Periodic arrangement of silica nanospheres assisted by amino acids. J. Am. Chem. Soc. 2006, 128 (42), 13664-13665.

(30) Yuan, J.-J.; Mykhaylyk, O. O.; Ryan, A. J.; Armes, S. P. Cross-linking of cationic block copolymer micelles by silica deposition. J. Am. Chem. Soc. 2007, 129 (6), 1717-1723.

(31) Tomczak, M. M.; Glawe, D. D.; Drummy, L. F.; Lawrence, C. G.; Stone, M. O.; Perry, C. C.; Pochan, D. J.; Deming, T. J.; Naik, R. R. Polypeptide-templated synthesis of hexagonal silica platelets. J. Am. Chem. Soc. 2005, 127 (36), 12577-12582. 
(32) Jakhmola, A.; Vecchione, R.; Guarnieri, D.; Belli, V.; Calabria, D.; Netti, P. A. Bioinspired Oil Core/Silica Shell Nanocarriers with Tunable and Multimodal Functionalities. Adv. Healthcare Mater. 2015, 4 (17), 2688-2698.

(33) Erni, P.; Dardelle, G.; Sillick, M.; Wong, K.; Beaussoubre, P.; Fieber, W. Turning coacervates into biohybrid glass: Core/shell capsules formed by silica precipitation in protein/polysaccharide scaffolds. Angew. Chem. 2013, 125 (39), 10524-10528.

(34) Wibowo, D.; Zhao, C.-X.; Middelberg, A. P. Interfacial Biomimetic Synthesis of Silica Nanocapsules Using a Recombinant Catalytic Modular Protein. Langmuir 2015, 31 (6), 19992007.

(35) Chen, Y.; Kang, E.-T.; Neoh, K.-G.; Greiner, A. Preparation of Hollow Silica Nanospheres by Surface - Initiated Atom Transfer Radical Polymerization on Polymer Latex Templates. Adv. Funct. Mater. 2005, 15 (1), 113-117.

(36) Zhao, W.; Lang, M.; Li, Y.; Li, L.; Shi, J. Fabrication of Uniform Hollow Mesoporous Silica Spheres and Ellipsoids of Tunable Size Through a Facile Hard-Templating Route. J. Mater. Chem. 2009, 19 (18), 2778-2783.

(37) Wibowo, D.; Yang, G.-Z.; Middelberg, A. P. J.; Zhao, C.-X. Non-Chromatographic Bioprocess Engineering of a Recombinant Mineralizing Protein for the Synthesis of Silica Nanocapsules. Biotechnol. Bioeng. 2016, n/a-n/a.

(38) Kasai, A.; Hayashi, T. I.; Ohnishi, H.; Suzuki, K.; Hayasaka, D.; Goka, K. Fipronil application on rice paddy fields reduces densities of common skimmer and scarlet skimmer. Sci. Rep. 2016, 6.

(39) Li, Z.-Z.; Wen, L.-X.; Shao, L.; Chen, J.-F. Fabrication of porous hollow silica nanoparticles and their applications in drug release control. J. Controlled Release 2004, 98 (2), 245-254.

(40) Mahony, D.; Cavallaro, A.; Mody, K.; Xiong, L.; Mahony, T.; Qiao, S.; Mitter, N. In vivo delivery of bovine viral diahorrea virus, E2 protein using hollow mesoporous silica nanoparticles. Nanoscale 2014, 6 (12), 6617-6626.

(41) Li, Z.-Z.; Xu, S.-A.; Wen, L.-X.; Liu, F.; Liu, A.-Q.; Wang, Q.; Sun, H.-Y.; Yu, W.; Chen, J.-F. Controlled Release of Avermectin from Porous Hollow Silica Nanoparticles: Influence of Shell Thickness on Loading Efficiency, UV-Shielding Property and Release. J. Controlled Release 2006, 111 (1), 81-88.

(42) Goscianska, J.; Olejnik, A.; Nowak, I.; Marciniak, M.; Pietrzak, R. Ordered Mesoporous Silica Modified with Lanthanum for Ibuprofen Loading and Release Behaviour. Eur. J. Pharm. Biopharm. 2015, 94, 550-558.

(43) Brinker, C. J. Hydrolysis and condensation of silicates: Effects on structure. Journal of Non-Crystalline Solids 1988, 100 (1), 31-50.

(44) Bobé, A.; Meallier, P.; Cooper, J.-F.; Coste, C. M. Kinetics and Mechanisms of Abiotic Degradation of Fipronil (Hydrolysis and Photolysis). J. Agric. Food Chem. 1998, 46 (7), $2834-$ 2839.

(45) Li, F. H.; Yao, K.; Lv, W. Y.; Liu, G. G.; Chen, P.; Huang, H. P.; Kang, Y. P. Photodegradation of Ibuprofen under UV-Vis Irradiation: Mechanism and Toxicity of Photolysis Products. Bull. Environ. Contam. Toxicol. 2015, 94 (4), 479-483.

(46) Kerwin, B. A.; Remmele, R. L. Protect from Light: Photodegradation and Protein Biologics. J. Pharm. Sci. 2007, 96 (6), 1468-1479.

(47) Samanta, S.; Kole, R.; Chowdhury, A. Photodegradation of Metsulfuron Methyl in Aqueous Solution. Chemosphere 1999, 39 (6), 873-879. 
(48) Burrows, H. D.; Santaballa, J.; Steenken, S. Reaction Pathways and Mechanisms of Photodegradation of Pesticides. J. Photochem. Photobiol., B 2002, 67 (2), 71-108.

(49) Zhou, S.; Wu, L. Transparent organic-inorganic nanocomposite coatings. Functional Polymer Coatings: Principles, Methods, and Applications 2015, 12, 1.

(50) Kristo, E.; Hazizaj, A.; Corredig, M. Structural changes imposed on whey proteins by UV irradiation in a continuous UV light reactor. J. Agric. Food Chem. 2012, 60 (24), 6204-6209. 
TABLE OF CONTENT GRAPHIC

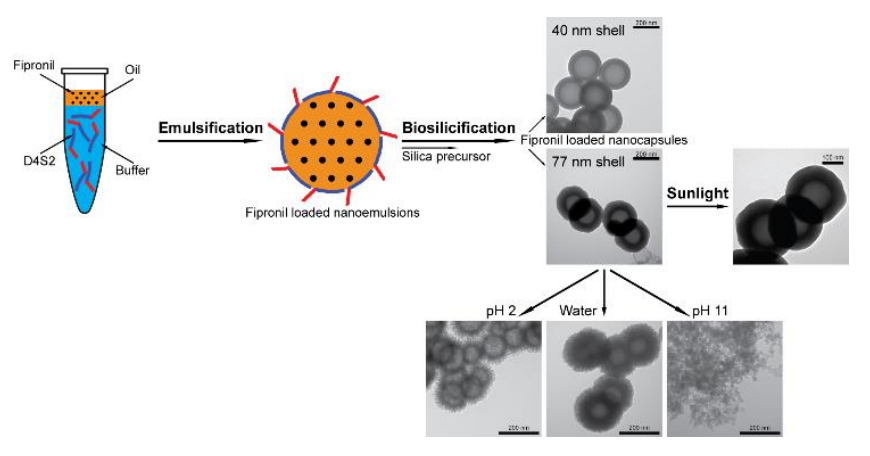

